\title{
AN EMPIRICAL MODEL FOR EARLY RESISTANCE CHANGES DUE TO ELECTROMIGRATION
}

\author{
J. NIEHOF, H. C. DE GRAAFF, A. J. MOUTHAAN and J. F. VERWEY \\ MESA Research Institute, University of Twente, Department of Electrical Engineering, Group ICE, \\ P.O. Box 217, 7500 AE Enschede, The Netherlands
}

(Received II August 1994; in revised form 1 December 1994)

\begin{abstract}
A new heuristic description for electromigration-induced early resistance changes is given. The basis is formed by two coupled partial differential equations, one for vacancies, and one for imperfections. These equations are solved numerically for a grain boundary bamboo structure. It is shown that this model is capable of simulating the typical effects as observed in early resistance change measurements. These early resistance changes are due to the redistributions of the vacancies and the generation of imperfections. Simulations are performed that closely match the measured resistance change curves.
\end{abstract}

\section{NOTATION}

$D$ diffusion constant $\left(\mathrm{cm}^{2} \mathrm{~s}^{-1}\right)$

$D_{0} \quad$ pre exponential constant $\left(\mathrm{cm}^{2} \mathrm{~s}^{-1}\right)$

$k$ Boltzmann's constant $\left(\mathrm{eV} \mathrm{K}^{-1}\right)$

$E_{\mathrm{b}} \quad$ bulk electromigration activation energy $(\mathrm{eV})$

$E_{\mathrm{gb}} \quad$ grain boundary activation energy (eV)

$E_{\mathrm{a}} \quad$ activation energy $(\mathrm{eV})$

$E_{\text {form }}$ vacancy formation energy (eV)

$E_{j} \quad$ current density-induced formation activation energy (eV)

$Z^{*} \quad$ effective charge number

e electronic charge (C)

$\rho_{\mathrm{b}} \quad$ bulk resistivity $(\mu \Omega \mathrm{cm})$

$\rho_{\mathrm{gb}} \quad$ grain boundary resistivity $(\mu \Omega \mathrm{cm})$

$\rho_{\mathrm{d}} \quad$ grain boundary dependent resistivity $(\mu \Omega \mathrm{cm})$

$R_{0} \quad$ initial stripe resistance $(\Omega)$

$G_{0}$ formation constant $\left(\mathrm{A}^{-1} \mathrm{~cm}^{-1} \mathrm{~s}^{-1}\right)$

$G_{10}$ formation constant $\left(\mathrm{A}^{-1} \mathrm{~cm}^{-1} \mathrm{~s}^{-1}\right)$

$m$ current density exponent

$\tau_{0} \quad$ time constant (h)

$a$ scaling constant $\left(\mu \mathrm{m}^{-2}\right)$

b scaling constant $\left(\mu \mathrm{m}^{-2}\right)$

$\gamma \quad$ vacancy resistivity $\left(\mu \Omega \mathrm{cm}(\text { at. } \% \mathrm{~V})^{-1}\right)$

$N_{0} \quad$ aluminium atomic density $\left(\mathrm{cm}^{-3}\right)$

$V_{\text {to }}$ pre-exponential constant $\left(\mathrm{cm}^{-3}\right)$

$V_{0}$ thermal equilibrium vacancy concentration $\left(\mathrm{cm}^{-3}\right)$

$N_{\text {i0 }}$ thermal equilibrium imperfection concentration $\left(\mathrm{cm}^{-3}\right)$

\section{INTRODUCTION}

Metallization failure in integrated circuits has been a problem in the semiconductor electronics industry ever since the advent of thin film technology. Advances in integrated-circuit technology from VLSI to ULSI cause a further down scaling of device dimensions and concurrently the width of the interconnection metallization decreases, resulting in increasing current densities. All this causes the reliability of ICs with respect to electromigration to decrease and it is becoming a major concern. In the past all electromi- gration models were an attempt to model the electromigration failure process as a whole. Models were published in various complexities, ranging from empirical models based on Black's equation, to atomistic models including grain boundary networks[1,2].

The work of Attardo[3] is one of the earliest attempts to incorporate both macroscopic design parameters, such as length and width, and microstructural features, such as grain size distribution, film orientation, and grain boundary character into one self-consistent model of electromigration. Attardo's model has been used as a starting point by other researchers[4-6] in their attempt to model electromigration. In those models time to failure is defined as the time it takes to reach a critical value of the vacancy density.

Other modelling work concentrates on the failure of a line, considering two time periods: the time to reach a critical vacancy density and then the time required for voids to grow to a critical size[2,7].

Recent reports of detailed direct observation of void formation and motion[8-10] have not been consistent with the widely held views on the subject. The electromigration process, from the first current stress until the ultimate stripe failure, has been observed to be a complex process of dynamic void behaviour $[9,10]$. The outcome of these observations is that none of the published models for dynamic void motion are consistent with the observations.

In spite of extensive efforts in the past years, the interpretation of some aspects of the electromigration phenomena remain controversial, and a complete, generally accepted physical model of the electromigration process does not yet exist.

Resistometric methods have been employed to study electromigration damage for some time now. Usually the sample resistance is monitored with a 
resistance change resolution in the order of percentages. Latcly a trend is found towards studying the earlier stages of the electromigration process by using more sensitive resistance change measurement techniques[11-15,17-19]. The problem is, when examining resistance changes with a higher resolution, that one has to make sure that the observed resistance changes are entirely electromigration-induced. Unwanted resistance changes, which lead to uncertainties in the interpretation, have to be eliminated in order to secure accurate and valid electromigrationinduced resistance change measurements.

The a.c.-bridge technique, first developed and applied by Lloyd and Koch[11], virtually eliminates the thermal effects and allows measurement of very small resistance changes (resolution in $\Delta R / R \sim 10^{-6}$ ). This method has been used by others to study electromigration-induced early resistance changes[13-16]. Sensitive measurements of electromigration induced resistance changes (using a.c.-bridge technique) have been shown to give predictable and reproducible results[11-15].

In this paper a new heuristic model will be presented, that has been developed in order to describe early phases in the electromigration process and their related resistance changes. The purpose of modelling these phases of the electromigration process is to develop a better understanding of the dynamics of vacancy behaviour and the related defect generation. The ultimate goal is then to use the model and the insights gained for failure prediction.

It has been shown that it is not possible to model electromigration-induced early resistance changes completely based on the dynamics of vacancies only (one-particle model)[20]. The one-particle model lacks the ability to describe a linear increase and remanence in resistance change that is generally observed[21].

There are a number of suggestions in literature pointing towards using more than one particle in a model. Lloyd and Koch[11] rule out the vacancy alone to be responsible for the resistance increase. In their work they report that vacancies alone cannot account for the observed behaviour and that a vacancy supersaturation would rapidly decay when the current is turned off. Furthermore the observed decay times in the order of hours above $400 \mathrm{~K}$ are not consistent with the decay of a vacancy supersaturation. They hold some vacancy supersaturation responsible for the resistance increases observed under current stress, suggesting that this vacancy supersaturation would create conditions where the nucleation of vacancy precipitates might occur. These defects, perhaps dislocation loops or prismatic defects, would be created as a function of the supersaturation and these defects would also contribute to the resistance increase. Stable defects can then account for the observed permanent change in resistance (remanence) when current stress is removed.
We have developed a new model describing electromigration-induced early resistance changes, also based on stable defects.

In Section 2 an intuitive physical basis, together with the description of our model is provided. The heart of the model consists of two coupled partial differential equations, one for vacancies, and one for resulting microstructural anomalies, called imperfections. These equations are solved numerically for a single grain boundary bamboo structure in Section 3 . In this section the functioning of the model is elucidated by examining the redistribution of the vacancies and imperfections and the concomitant change in resistance. For model verification purposes in Section 4 model calculations are compared with experimentally obtained resistance change curves. Then in Section 5 the results from the experimental verification are discussed.

\section{THE VACANCY/IMPERFECTION MODEL}

In a conductor under severe electric current stress not all the metallization atoms contribute to the electromigration process. The modelling equations should therefore concentrate on those particles, that play an active role in the electromigration process. The set of defects, dislocation loops or any other but vacancies increasing the scattering of electron, are referred to as imperfections here. So in this model the "imperfection" stands for a number of possible defects, a projection of the accumulation of damage, in the microstructure and most likely occurring at or near grain boundaries. The exact physical nature of the imperfections is not explicitly stated here and would require further materials research. For a correct description of the resistance change behaviour this second phenomenon seems necessary and there is enough indication to make the assumption acceptable. Therefore we propose the following model:

The particles that play an active role in the electromigration process are generated from the metal lattice: vacancies and imperfections. The generated vacancies are mobile and move under the influence of the driving force which is proportional to the applied electric field. When a vacancy is formed the number of scattering sources increases due to both the vacancy and the concomitant damage to the structure which we refer to as an imperfection. The process might be considered as the formation of vacancies generating stress, which in turn induces damages to the structure (imperfections). The imperfections are considered to be immobile (the structural changes caused by the vacancy formation do not move) and to remain at the position of their formation. We have assumed the growth rate of imperfections to be linear proportional to the net formation of vacancies. This also implies that the imperfection concentration can decrease with the annihilation of vacancies. We can distinguish three mechanisms determining the shape 
of the solution: diffusion $(\nabla V)$, electrotransport (field-term) and formation/annihilation.

Transport of vacancies will occur when a driving force is present. The flux ( $\mathbf{J})$ of vacancies $(V)$ under the influence of an applied electric field $(E)$ is given by:

$$
\mathbf{J}=-D \nabla V+\frac{V D}{k T} Z^{*} e \mathbf{E}
$$

$Z^{*}$ is known as the effective valence, which is an electromigration term representing the strength and the direction $\left(Z^{*}>0\right.$ for vacancy movement in $\mathrm{Al}$ and its alloys) of the electromigration driving force. The total vacancy flux $(J)$ consists of an electric-field part and of a diffusion part. The diffusion coefficient $D$ is thermally activated:

$$
D=D_{0} \exp \left(\frac{-E_{\mathrm{a}}}{k T}\right),
$$

where $E_{\mathrm{a}}$ is the activation energy, $D_{0}$ a pre-exponential factor.

Changes in the vacancy and imperfection density concentrations in time can be caused by formation, annihilation, and by divergences in the vacancy flux. The continuity equations are:

for vacancies:

$$
\frac{\partial V}{\partial t}=-\nabla \cdot \mathbf{J}-\frac{V-V_{0}}{\tau_{0}}+G(j)
$$

and for imperfections:

$$
\frac{\partial N_{\mathrm{i}}}{\partial t}=-\frac{V-V_{0}}{\tau_{0}}+G(j),
$$

where $N_{\mathrm{i}}$ concentration of imperfections $\left(\mathrm{cm}^{-3}\right), V$ concentration of vacancies $\left(\mathrm{cm}^{-3}\right), \mathbf{J}$ vacancy flux $\left(\mathrm{cm}^{-2} \mathrm{~s}^{-1}\right), G(j)$ current density-induced generation term $\left(\mathrm{cm}^{-3} \mathrm{~s}^{-1}\right), \tau_{0}$ average vacancy lifetime (s).

In any metallization there is always a thermal concentration of vacancies and some initial imperfection concentration. For the vacancies the thermal background vacancy concentration $V_{0}$ is given by:

$$
V_{0}=V_{\mathrm{t} 0} \exp \left(\frac{-E_{\mathrm{form}}}{k T}\right),
$$

where $E_{\text {form }}$ is the activation energy for vacancy formation, $T$ is the ambient temperature, $V_{\text {to }}$ is a constant. At thermal equilibrium and without any external disturbances, the thermal imperfection concentration is $N_{\text {i0 }}$.

As mentioned before, the growth rate of imperfections is supposed to be proportional to the net formation of vacancies; for reasons of simplicity we have chosen the proportionality constant to be equal to one.

A second assumption in our model is a current density-induced formation term, $G(j)$, according to Liew[22,23]:

$$
G(j)=G_{0}|j|^{m},
$$

where $G_{0}$ and $m$ are constants.
The absolute sign on the current density is used because the formation is considered not to depend on the direction of the electron flow. This current density-induced formation term appears to be essential in order to describe the resistance change curves with the correct time constant and magnitude: without current density-induced formation the time constant is too short[11,21] and the magnitude of the resistance change is too small[20,21]. Although essential, we will show in Section 3 that the current density-induced formation is small.

\subsection{Simulation structure}

In a purely homogeneous stripe of aluminium in thermal equilibrium, the concentration of vacancies equals the concentration of imperfections at any time and at any position in this stripe: $V_{0}=N_{\mathrm{i} 0}$. When applying current stress in this simple case, there is no divergence in the vacancy flux (no grain boundary): $\nabla \cdot \mathbf{J}=0$. Thus it follows from eqns (3) and (4) that $\partial N_{\mathrm{i}} / \partial t=\partial V / \partial t$, and that the concentration of vacancies will always be equal to the concentration of imperfections at any time and at any position within the stripe.

The influence of grain boundaries on the electromigration mass transport at temperatures ranging from $160-200^{\circ} \mathrm{C}$ is almost entirely due to the large difference (several orders in magnitude) in the diffusion constants for grain boundary and bulk. We have modelled, rather arbitrarily, but in order to prevent convergence problems in the numerical calculations, the activation energy of the diffusion constant by the following equation:

$$
E_{\mathrm{a}}=E_{\mathrm{b}}+\left(E_{\mathrm{gb}}-E_{\mathrm{b}}\right) \mathrm{e}^{-a x^{2}},
$$

where $x$ is the distance from the centre of the grain boundary, and $a$ is a scaling factor. For the electrical resistivity we also use an exponential dependence on the grain boundary structure:

$$
\rho_{\mathrm{d}}=\rho_{\mathrm{b}}+\left(\rho_{\mathrm{gb}}-\rho_{\mathrm{b}}\right) \mathrm{e}^{-b x^{2}},
$$
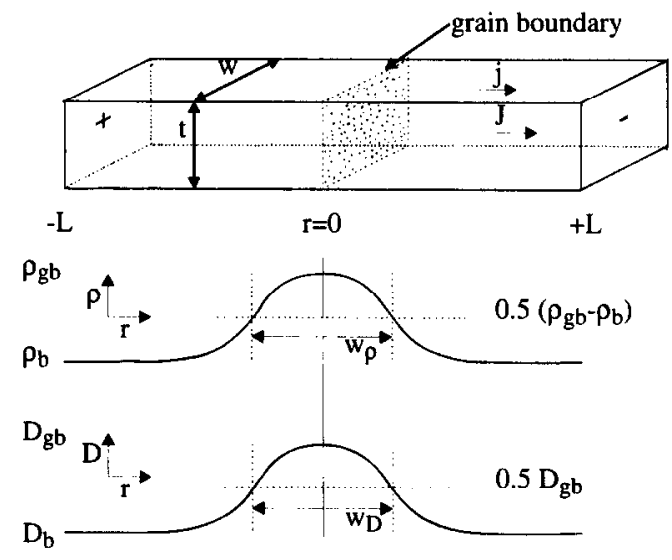

Fig. 1. Stripe with one grain boundary (bamboo structure), with the transitions in resistivity and diffusion constant. 
where $\rho_{\mathrm{b}}$ is the resistivity inside the grain, and $\rho_{\mathrm{gb}}$ is the resistivity in the centre of the grain boundary.

The steepness of these transitions can be controlled by the parameters $a$ and $b$. The scaling parameters $a$ and $b$ should be set independently in such a way that the effective grain boundary width of a bamboo structure has the same magnitude for the diffusivity and resistivity ( $w_{\rho} \approx w_{\mathrm{D}}$, see Fig. 1).

The effective width of the grain boundary in the bamboo structure in Fig. 1 is defined from the diffusion constant $D=0.5 D_{\mathrm{gb}}$ with the help of eqns (2) and (7):

$$
w_{\mathrm{D}}=2 \sqrt{\frac{-1}{a} \ln \left(1+\frac{k T \ln (0.5)}{\left(E_{\mathrm{b}}-E_{\mathrm{gb}}\right)}\right)} .
$$

The effective grain boundary width for the resistivity value $\rho=\rho_{\mathrm{b}}+0.5\left(\rho_{\mathrm{gb}}-\rho_{\mathrm{b}}\right)$ becomes with eqn (8):

$$
w_{\rho}=2 \sqrt{\frac{1}{b} \ln (2)} .
$$

The scaling parameters $a$ and $b$ should be chosen such that $w_{\mathrm{D}}=w_{\rho}$. Then from eqns (9) and (10) it follows:

$$
b=20 a .
$$

The resistivity in the grain boundary is higher than inside the grain $\left(\rho_{\mathrm{gb}}>\rho_{\mathrm{b}}\right)$ due to the distorted lattice in the grain boundary region. This implies a higher local electric field which enhances the atomic transport in the grain boundary. So in the grain boundaries both a higher diffusivity (lower activation energy) and a higher driving force exist.

The bamboo structure is used because it is considered to be the most simple representation of a thin film conductor; the grain boundary is the cause for the flux divergences and the concomitant redistribution of vacancies (and imperfections). A structure with more than one grain boundary perpendicular to the direction of current flow, or even whole grain boundary networks are also possible simulation structures. More complex grain boundary networks can be considered as the series and parallel connection of bamboo and triple point units. The resistance change characteristics for the bamboo and triple point structure being calculated, the total stripe resistance change curve is then given by the series and parallel resistances of these basic elements.

\subsection{Resistance model}

Electron mobility depends on the scattering of electrons. Both a vacancy and an imperfection will increase electron scattering and give a decrease in electron mobility. According to Matthiesen's rule these scattering sources should be added. Therefore, the resistivity due to increased scattering is modelled by:

$$
\begin{aligned}
\rho(x, t)= & \rho_{\mathrm{d}}(x)\left\{1+\xi\left[\left(V(x, t)-V_{0}\right)\right.\right. \\
& \left.\left.+\left(N_{\mathrm{i}}(x, t)-N_{\mathrm{i} 0}\right)\right]\right\},
\end{aligned}
$$

where:

$$
\xi=\frac{\gamma}{\rho_{\mathrm{d}} N_{0}} .
$$

The aluminium atom density, $N_{0}$, in the grain boundary is, because of the distorted lattice, less than in the grains. The behaviour of the resistivity, $\rho_{d}$, is described in the previous section. And for the vacancy resistivity, $\gamma$ (change in resistivity per atomic percent vacancies): an extra vacancy will have less influence in the already much more scattering-source rich grain boundary than in the cleaner gain regions. So the value of $\xi$ is influenced by counteracting effects; for convenience it is assumed here that $\xi=\gamma / \rho_{\mathrm{d}} N_{0}$ is constant and that the grain boundary influence in $\xi$ is levelled out, even though $\gamma, N_{0}$ and $\rho_{\mathrm{d}}$ are positiondependent[20]. Then the relative resistance change is given by:

$$
\begin{aligned}
\frac{\Delta R}{R_{0}} & = \\
& \frac{\xi \int_{0}^{L} \rho_{\mathrm{d}}(x)\left\{\left(V(x, t)-V_{0}\right)+\right.}{\left.\left(N_{\mathrm{i}}(x, t)-N_{\mathrm{i} 0}\right)\right\} \mathrm{d} x}
\end{aligned}
$$

where $R_{0}$ is the initial stripe resistance. So, eqns (1), (3), (4), (12) and (14) model the early resistance changes of the structure due to electromigration by means of the formation and redistribution of vacancies and imperfections. An overview of all model parameters is given in the notation.

\subsection{Two essential features}

Sensitive resistance change measurements show a fast increase in $\Delta R / R$ as soon as current is applied[11-16]. In terms of our model the fast increase after applying the current is modelled by the current density-induced excess formation of vacancies and imperfections $G(j)$ in eqn (6).

This excess formation gives rise to an exponential change in both the vacancy and imperfection concentrations. In the simple case, when there is no divergence in the vacancy flux $(\nabla \cdot \mathbf{J}=0)$, the vacancy concentration will rise from thermal equilibrium value $V=V_{0}$ to $V=V_{0}+\tau_{0} G(j)$. The imperfection concentration shows an equal rise.

The essential, crucial difference of this model as compared to other, previous models is that this model keeps track of both the mobile (vacancies) and the immobile (imperfections) particles, by using two continuity equations. When concentrating on just the mobile particles, and using one continuity equation only, we will find after some time a steady-state situation in the mobile particle distribution. In our model we find a steady state situation for the vacancies: 


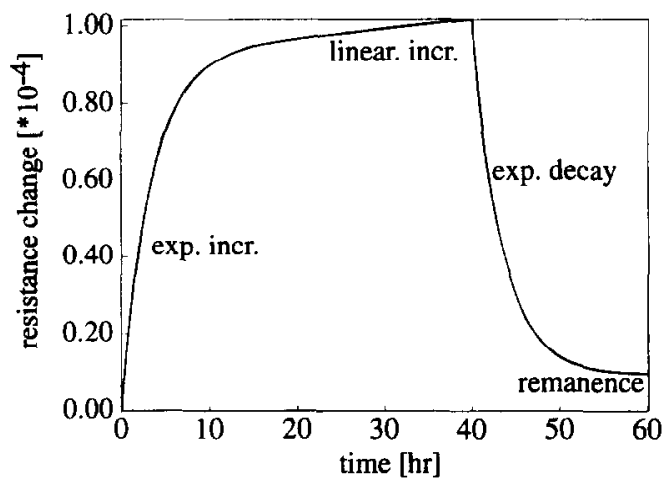

Fig. 2. Resistance change due to electromigration as calculated by the model. Current stress is applied from $t=0$ to $40 \mathrm{~h}$.

$$
\frac{\partial V}{\partial t}=0=-\nabla \cdot \mathbf{J}-\frac{V-V_{0}}{\tau_{0}}+G(j),
$$

but for the imperfections:

$$
\frac{\partial N_{\mathrm{i}}}{\partial t}=-\frac{V-V_{0}}{\tau_{0}}+G(j)=\nabla \cdot \mathbf{J} \neq 0 .
$$

In Sections 3 and 4 we will show that both features, the continuing change in the imperfection distribution and the use of a small current density-induced formation, are essential for fitting the experimentally found early resistance changes.

\section{SIMULATION ON A SINGLE GRAIN BOUNDARY}

The vacancy/imperfection model as presented above is implemented in our $2 \mathrm{D}$ process and device simulator TRENDY[25]. TRENDY solves the system of the two coupled partial differential equations on a finite difference grid. Simulations with the model are run on a HP 9000-750 workstation.

First simulations were performed on a fictitious 1D stripe of aluminium with a single grain boundary perpendicular to the direction of current flow (bamboo structure) as shown in 3D in Fig. 1, to illustrate the typical behaviour of the resistance change. The behaviour of the structure is simulated for a total of $60 \mathrm{~h}$. During the first $40 \mathrm{~h}$ a current density of

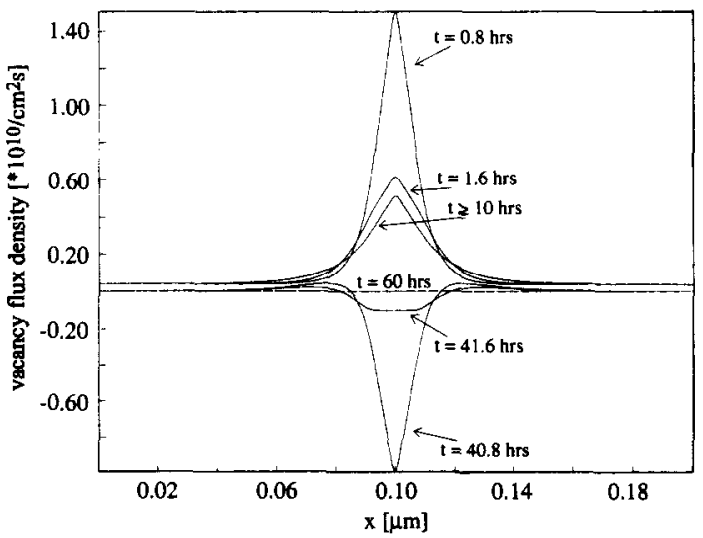

Fig. 3. Vacancy flux density along the stripe.

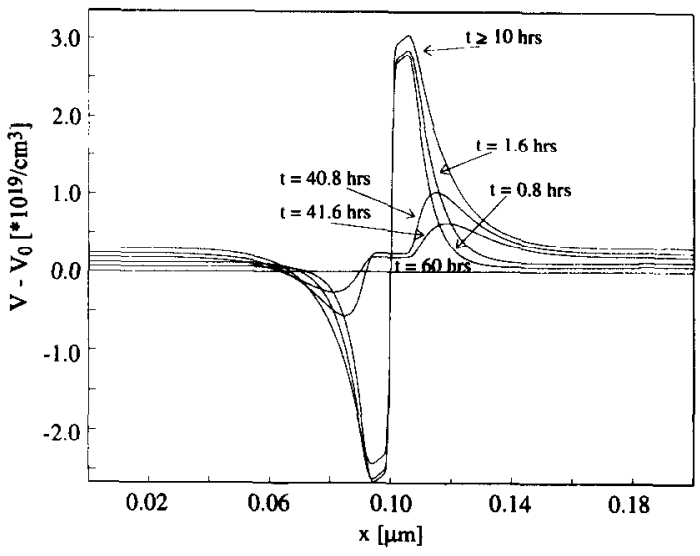

Fig. 4. Vacancy distribution in time.

$1 \mathrm{MA} / \mathrm{cm}^{2}$ is forced through the stripe. From $40-60 \mathrm{~h}$ the recovery behaviour of the stripe after removal of current stress is studied.

As the electric current density is from left to right, as depicted in Fig. 1, the major part of the total vacancy flux is in that direction. In the following Figs 3-5 the middle of the grain boundary is at $x=0.1 \mu \mathrm{m}$.

Figure 2 gives the simulated resistance change in time according to the model. In the resistance change curve under current stress $(t=0-40 \mathrm{~h})$ we can point out two regions: first, fast exponential increase and then growth at a linear rate.

The resistance change after removal of current stress $(t=40-60 \mathrm{~h})$ decays exponentially and saturates at a non-zero level: a permanent resistance change remains as a witness of the permanent damage caused by the $40 \mathrm{~h}$ current stress[11,26]. The model parameters as used for these calculations are given in Table 1.

Except for the resistivity and the activation energy [eqns (7) and (8)] all model parameters are constant throughout the simulation structure.

Resistance relaxation as calculated by the model occurs with a characteristic time close to the $\tau_{0}$ in the model. From considering measured resistance change

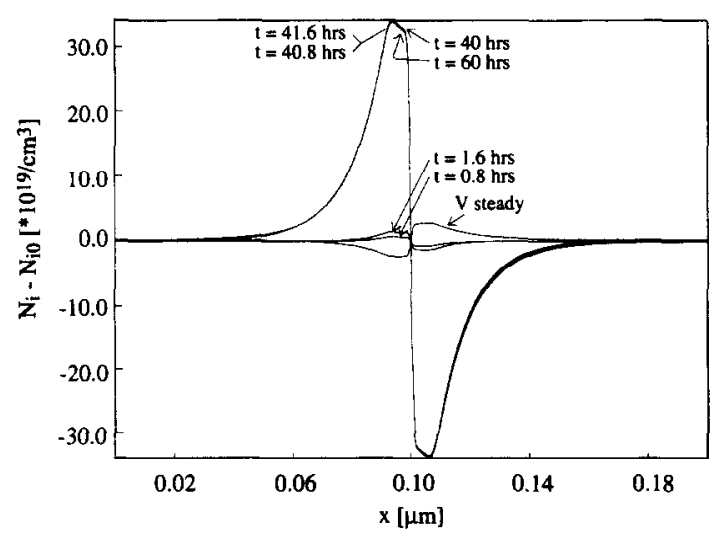

Fig. 5. Imperfection distribution in time. 


\begin{tabular}{|c|c|c|c|c|c|}
\hline $\begin{array}{l}T \\
j \\
N_{0} \\
Z^{*}\end{array}$ & $\begin{array}{l}473 \mathrm{~K} \\
1 \mathrm{MA} / \mathrm{cm}^{2} \\
6.026 e 22 \mathrm{at} . \mathrm{cm}^{3} \\
20\end{array}$ & $\begin{array}{l}D_{0} \\
E_{\mathrm{b}} \\
E_{\mathrm{gb}} \\
\rho_{\mathrm{b}} \\
\rho_{\mathrm{gb}}\end{array}$ & $\begin{array}{l}0.1 \mathrm{~cm}^{2} / \mathrm{s} \\
1.4 \mathrm{eV} \\
0.6 \mathrm{eV} \\
2.45 \mu \Omega \mathrm{cm} \\
250 \mu \Omega \mathrm{cm}\end{array}$ & $\begin{array}{l}m \\
G_{0} \\
\tau_{0} \\
a \\
b\end{array}$ & $\begin{array}{l}1 \\
2.8 e 8 \mathrm{~A} \cdot \mathrm{cm}^{-1} \mathrm{~s}^{\prime} \\
3 \mathrm{~h} \\
6 e 4 \mu \mathrm{m}= \\
1.2 e 6 \mu \mathrm{m}^{2}=\end{array}$ \\
\hline
\end{tabular}

curves we have chosen to use $\tau_{0}=3 \mathrm{~h}[13]$. For the thermal concentration of vacancies and imperfections we have chosen, rather arbitrarily, $V_{0}=N_{\mathrm{i} 0}=4$ at. $\%$. In literature values for $V_{0}$ ranging from 0.01 to 20 at. $\%$ are used[27,28]. Within this range for $V_{0}$ no considerable changes are found in the shape of the calculated resistance change curve compared to Fig. 2.

More important for the resistance change is the ratio $\tau_{0} G(j)$ to $V_{0}$ and $N_{\mathrm{i} 0}$. The exponential part of the resistance increase is mainly caused by the current density-induced rise in vacancy and imperfection concentration from $V_{0}$ to $V_{0}+\tau_{0} G(j)$. From experiments the exponential resistance change is estimated to be in the order of $10^{-4}$. Through eqn (14) it follows that the relative resistance change, caused by the rise in vacancy and imperfection level, is $2 \xi \tau_{0} G(j)$. According to the arguments in Section 2.2 and since $\gamma$ and $\rho_{\mathrm{d}}$ are in the same order of magnitude, $\xi=\gamma /\left(\rho_{\mathrm{d}} N_{0}\right)=1 / N_{0}$ is used. Then from $2 \xi \tau_{0} G(j) \sim 10^{-4}$ and using $m=1[16,29], \quad \xi=1 / N_{0} \quad$ and $j=1 \mathrm{MA} / \mathrm{cm}^{2}, \quad$ it follows that $\mathrm{G}_{0}=2.8 e 8 \mathrm{~A}^{-1} \mathrm{~cm}^{-1} \mathrm{~s}^{-1}$. This makes $\tau_{0} G(j) \sim 10^{-3} V_{0}$; although small, the current densityinduced formation $G(j)$ is essential. Without it there will be hardly any exponential resistance increase $\left(\Delta R / R_{0} \sim 10^{-6}[20]\right)$. For the width of this single grain boundary $w_{\mathrm{gb}}=1.5 \mathrm{~nm}$ is used[24]. This requires setting $a=6 e 4 \mu \mathrm{m}^{-2}$ and $b=1.2 e 6 \mu \mathrm{m}^{-2}$.

For the resistivity in the grains the Al bulk resistivity is used: $\rho_{\mathrm{b}}=2.45 \mu \Omega \mathrm{cm}$. From fitting model calculations on experimentally obtained resistance change curves the resistivity at the grain boundary, $\rho_{\mathrm{gb}}$, was found to be approximately two orders in magnitude higher than $\rho_{\mathrm{b}}$. Therefore $\rho_{\mathrm{gb}}=250 \mu \Omega \mathrm{cm}$ is used. Note that $w_{\mathrm{gb}}$ and $\rho_{\mathrm{gb}}$ both describe the effective resistance of the grain boundary region and an increase in one can compensate a decrease in the other. For all other parameters in Table 1 generally accepted values are used. The vacancy flux $(J)$ in this bamboo structure is given in Fig. 3 .

The vacancy flux is higher in the grain boundary region because of the lower activation energy and therefore higher atom mobility in the grain boundary, and the peak in the electric field caused by the higher resistivity of the grain boundary [refer to eqn (1)]. The behaviour of the vacancy flux in time can be explained by observing the redistribution of the vacancies as given in Fig. 4. The changes are almost entirely situated at and near the grain boundary.

\subsection{Exponential resistance increase}

The divergence in the transport of vacancies, caused by the grain boundary, gives rise to a redistribution of vacancies: depletion on the anode side and accumulation on the cathode side of the grain boundary. Immediately after applying the current stress the electric field component in eqn (1) is observed in the vacancy flux density distribution $\left(J\right.$ at $\left.t=0.8 \mathrm{~h}<\tau_{0}\right)$ causing a fast redistribution of vacancies. This flux is more and more counteracted by the diffusion component ( $J$ at $t=1.6 \mathrm{~h}$ ), finally resulting in a steady state situation $(J$ for $t \geqslant 10 \mathrm{~h})$. The plots for the vacancy flux and vacancy distribution both indicate this steady state. In the regions outside the grain boundary, close to the contacts, the plots for the vacancy distribution show the change in concentration, caused by the current density-induced excess formation $G(j)$, from $V=V_{0}$ to $V=V_{0}+\tau_{0} G(j)$ and with a time constant $\tau_{0}$.

\subsection{Linear resistance increase}

The behaviour of the imperfection distribution under current stress is given in Fig. 5. To elucidate the difference in behaviour between vacancies and imperfections, the vacancy distribution at steady state is also shown in this figure ( $V$ steady). The imperfection distribution does not settle at a steady-state, because in the steady-state situation for the vacancies the divergence in the vacancy flux balances the total formation (formation-annihilation); refer to eqns (15) and (16). This implies that the vacancies that are generated on the anode side, move to the cathode side of the grain boundary where they annihilate. As the vacancies are swept across the grain boundary the imperfections remain behind. Thus at the anode side of the grain boundary we have an effective formation of imperfections and vacancies, whereas on the cathode side they annihilate. All this means that on the anode side of the grain boundary the imperfection concentration continues to grow because of the constant formation. On the cathode side the annihilation results in a continuing depletion of imperfections.

So, the imperfection distribution continues to change at a linear rate as long as current stress is applied, as does the resistance change.

Thus in our model we do find a steady-state situation as other models do, but on top of that we find a continuing increase of electromigration damage in that the imperfections continue to accumulate on the anode side and continue to deplete on the cathode side of the grain boundary. The imperfection distribution can physically be interpreted as a reflection of a change in the grain boundary structure in the film. Thus the resistance changes are attributed to remanent structural changes in the grain boundary structure. 


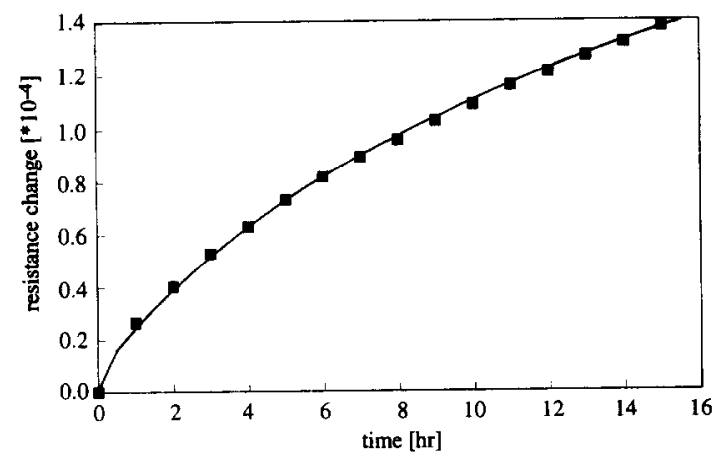

Fig. 6. Resistance change in time for $T=459 \mathrm{~K}$ and $j=1 \mathrm{MA} / \mathrm{cm}^{2}$. The solid curve shows the simulated resistance change. The markers indicate measured data points[30].

\subsection{Exponential resistance decay}

After removing the applied current, a vacancy flux across the grain boundary is found in the opposite direction ( $J$ at $t=40.8 \mathrm{~h}$ in Fig. 3): now the gradient in the vacancy distribution is the only driving force. At the grain boundary, where the vacancy mobility is high, this movement is fast, and here the vacancy distribution is soon becoming flat ( $V$ at $t=40.8$ and $41.6 \mathrm{~h}$ in Fig. 4).

Somewhat further away from the centre of the grain boundary the vacancy mobility is much less. Here formation and annihilation are the dominant processes in the redistribution (formation on the anode side and annihilation on the cathode side of the grain boundary). Finally the vacancy distribution has fallen completely back to its thermal equilibrium level $V=V_{0}(V$ at $t=60 \mathrm{~h})$.

\subsection{Remanence of resistance change}

Apart from the shift (close to the contacts) from $N_{\mathrm{i}}=N_{\mathrm{i} 0}+\tau_{0} G(j)$ to $N_{\mathrm{i}}=N_{\mathrm{i} 0}$, the imperfection distribution changes only slightly after removal of the current stress ( $N_{\mathrm{i}}$ at $t=40,40.8,41.6$ and $60 \mathrm{~h}$ in Fig. 5). There is not much time for annihilation, because of the initially fast, gradient-induced

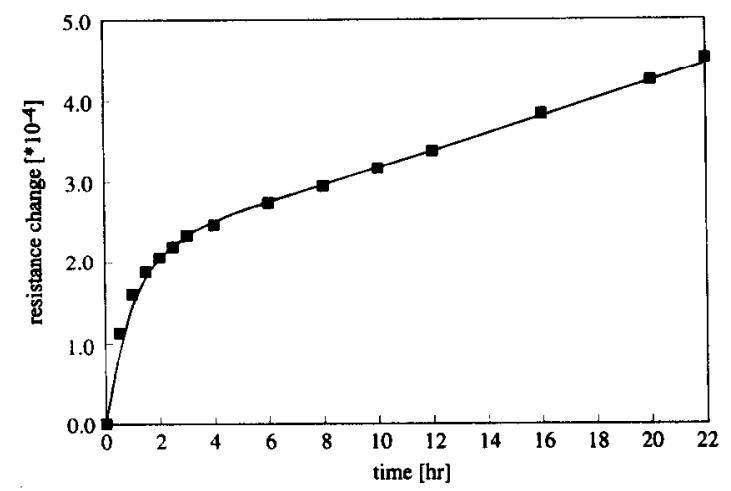

Fig. 7. Resistance change in time for $T=463 \mathrm{~K}$ and $j=0.83 \mathrm{MA} / \mathrm{cm}^{2}$. The solid curve shows the simulated resistance change. The markers indicate measured data points, taken from [13].

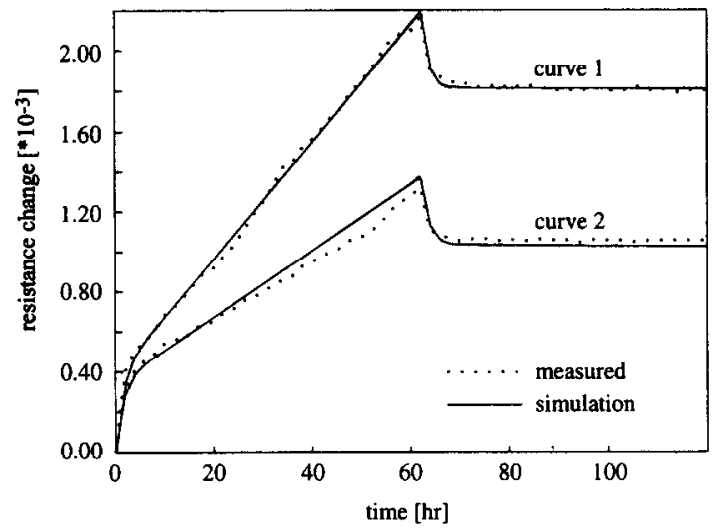

Fig. 8. Resistance change in time for $T=513 \mathrm{~K}$ and $j=2.0 \mathrm{MA} / \mathrm{cm}^{2}$. The solid curves show the simulated resistance changes. Measurement data taken from [26]. Current stress was applied from $t=0$ to $62 \mathrm{~h}$.

redistribution of vacancies as soon as current stress is removed. After some time the vacancy distribution has fallen completely back to the thermal equilibrium level $V=V_{0}$, then $\partial V / \partial t=0$. Because there are no driving forces anymore, the vacancy flux is also zero, resulting in $\partial N_{\mathrm{i}} / \partial t=0$. This means that the imperfection distribution does not change further and that the remaining magnitude of the imperfection peaks at the grain boundary is a measure of the amount of electromigration stress the stripe has experienced $\left(N_{\mathrm{i}}\right.$ at $t=60 \mathrm{~h}$ ). It also means that the resistance change, accumulated during the current stress, cannot be fully annulled afterwards: a remanence of resistance change.

\subsection{Resistance change curve control parameters}

Since the exponential rise of the resistance change is mainly caused by the current density-induced rise in vacancies and imperfections, the model parameters controlling this part of the curve are $G_{0}, m$ [refer to eqn (6)] and $\tau_{0}$. The behaviour of the linear region of the resistance change curve is determined by the equilibrium between the divergence in the vacancy

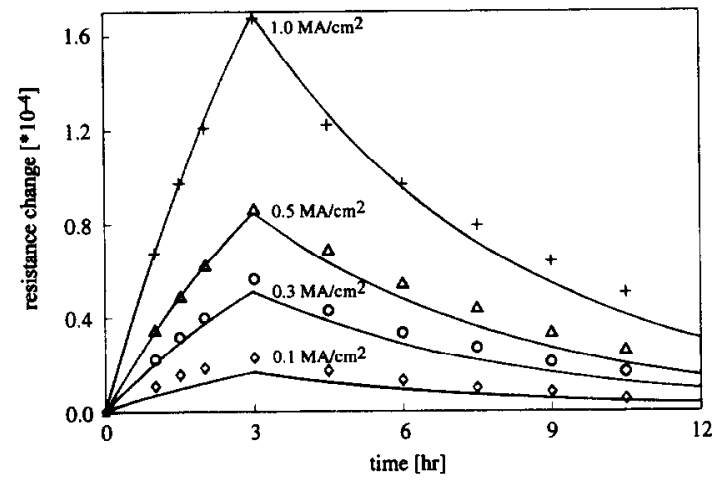

Fig. 9. Resistance change in time as a function of current density at $T=459 \mathrm{~K}$. The solid curves are the simulated resistance changes for $j=1,0.5,0.3$ and $0.1 \mathrm{MA} / \mathrm{cm}^{2}$. The markers indicate measured data points[30] 
flux and formation/annihilation; see eqn (18). Therefore the model parameters controlling this part of the resistance change, without interfering with the exponential resistance change part, are all parameters influencing the vacancy flux [refer to eqn (1)]: $j, \rho_{g b}$ and $w_{\mathrm{gb}}$.

Thus in summary, the exponential resistance change regions are formation and annihilation controlled, whereas the linear increase region is under control of the vacancy flux (through its divergence, determined by the grain boundary parameters).

\section{COMPARISON WITH MEASUREMENTS}

The simulations have been performed on the bamboo unit structure (refer to Fig. 1). We used this single grain boundary structure, because using more complex grain boundary structures, like more bamboo structures in series or triple points, will not seriously change the shape of the relative resistance change curve[20]. For the model checking we have used measured resistance change curves taken from the literature[13,26,30,31].

The model parameters are adjusted so that the resulting resistance change curve fits as close as possible to the measured curve.

\subsection{Resistance change at constant current density and temperature}

Figure 6 shows the result of a simulation of electromigration-induced resistance change over $16 \mathrm{~h}$ as measured on an Al stripe.

The model parameters used for this calculation are the same as used for the simulation on the single grain boundary structure in Table 1, except for:

$$
\begin{aligned}
\tau_{0} & =2.5 \mathrm{~h}, G_{0}=2.75 e 8 \mathrm{~A}^{-1} \mathrm{~cm}^{-1} \mathrm{~s}^{-1}, \\
\rho_{\mathrm{gh}} & =900 \mu \Omega \mathrm{cm} .
\end{aligned}
$$

The result of a simulation with the model of a resistance change curve of an $\mathrm{Al} / \mathrm{Si}$ sample over $22 \mathrm{~h}$ is given in Fig. 7. The model parameters that were changed here, as compared to the initial settings given in Table 1 are:

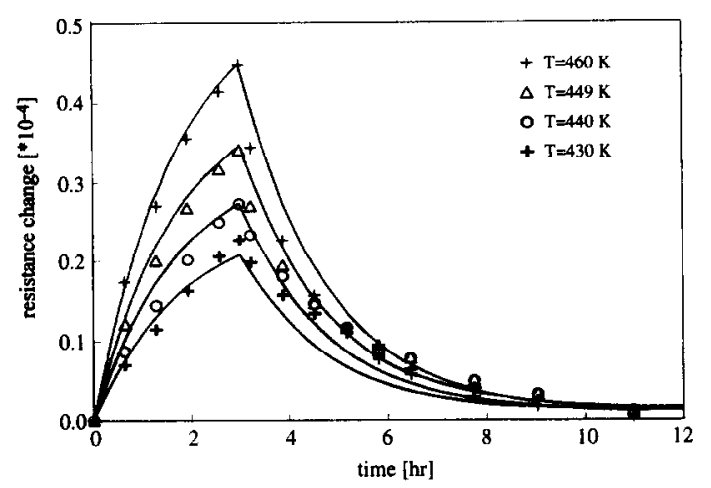

Fig. 10. Resistance change in time as a function of temperature at $j=3 \mathrm{MA} / \mathrm{cm}^{2}$ for pure Al. The solid curves are the simulated resistance changes for temperatures $460,449,440$ and $430 \mathrm{~K}$. The markers indicate measured data points[31].

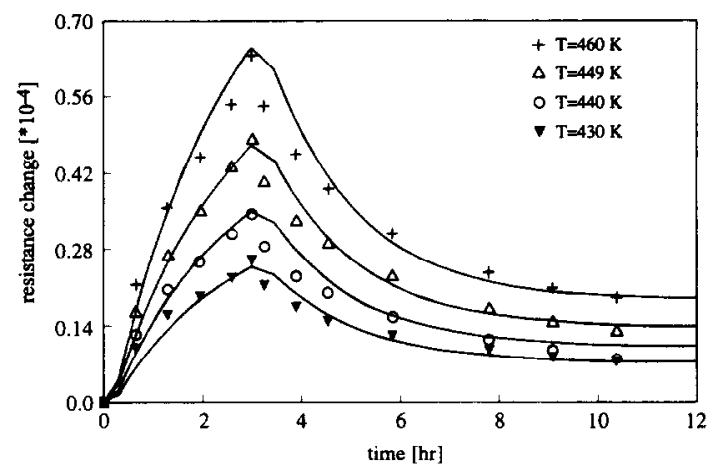

Fig. 11. Resistance change in time as a function of temperature at $j=3 \mathrm{MA} / \mathrm{cm}^{2}$ for $\mathrm{Al} / \mathrm{Si}$. The solid curves are the simulated resistance changes for temperatures $460,449,440$ and $430 \mathrm{~K}$. The markers indicate measured data points[31].

$$
\begin{aligned}
\tau_{0} & =0.75 \mathrm{~h}, G_{0}=3.2 e 9 \mathrm{~A}^{-1} \mathrm{~cm}^{-1} \mathrm{~s}^{-1}, \\
\rho_{\mathrm{gb}} & =900 \mu \Omega \mathrm{cm} .
\end{aligned}
$$

Figure 8 shows the result of simulations with the model on measurements by Scorzoni on $\mathrm{Al} / \mathrm{Si}$ samples[26]. The model parameters adjusted to match these curves are:

$$
\text { curve } \begin{aligned}
& 1: \tau_{0}=0.69 \mathrm{~h}, G_{0}=4.9 e 9 \mathrm{~A}^{-1} \mathrm{~cm}^{-1} \mathrm{~s}^{-1}, \\
& \rho_{\mathrm{gb}}=1095 \mu \Omega \mathrm{cm} . \\
& \text { curve 2: } \tau_{0}=0.69 \mathrm{~h}, G_{0}=4.3 e 9 \mathrm{~A}^{-1} \mathrm{~cm}^{-1} \mathrm{~s}^{-1}, \\
& \rho_{\mathrm{gb}}=850 \mu \Omega \mathrm{cm} .
\end{aligned}
$$

The curves in Fig. 8 are measured on two similar samples, under the same stressing conditions. The difference in experimental resistance change behaviour translates into two model parameters, $G_{0}$ and $\rho_{\mathrm{gb}}$. The parameter $\tau_{0}$, which controls the speed of exponential resistance change, is the same for both curves. The amount of remanence of resistance change calculated by the model is directly related to the linear resistance increase part, refer to Section 3.4. The curves in Fig. 8 show that this relation between the remanence and the linear part of the resistance change as anticipated by the model suits very well the experiments. For the curves in Figs 6-8 the parameter $\tau_{0}$ is adjusted to match the speed of the exponential increase, and the current density-induced excess formation is adjusted, through $G_{0}$, to account for the height of the exponential resistance change. The rate of linear resistance is controlled through $\rho_{\mathrm{gb}}$.

\subsection{Resistance change as function of current density}

Figure 9 gives resistance change curves, on pure $\mathrm{Al}$ samples, at $T=459 \mathrm{~K}$ for four current densities[30]. Both measured data points and calculated curves are given. The parameters change, compared to the parameter set given in Table 1, to fit the curve for $j=1 \mathrm{MA} / \mathrm{cm}^{2}$ are here:

$$
\tau_{0}=5 \mathrm{~h}, G_{0}=6.93 e 8 \mathrm{~A}^{-1} \mathrm{~cm}^{-1} \mathrm{~s}^{-1} \text {. }
$$


The curves for the other current densities were obtained without changing these parameters. For the current density exponent in the excess formation term $G(j)$, [refer to eqn (6)] $m=1$ is used. Thus from the model calculations on these measurements it follows that the current-induced excess formation depends more or less linearly on the applied current density. The results in Fig. 9 show the capability of the model in describing resistance curves as a function of current density.

\subsection{Resistance change as function of temperature}

To accommodate for the temperature dependence of the resistance changes, the constant $G_{0}$ in the current density-induced excess formation term [refer to eqn (6)] is slightly modified to incorporate an activation energy $\left(E_{j}\right)$ for the temperature dependence.

$$
G_{0}=G_{\mathrm{t} 0} \exp \left(\frac{-E_{j}}{k T}\right) .
$$

Figure 10 gives resistance change curves, on pure $\mathrm{Al}$ samples, at $j=3 \mathrm{MA} / \mathrm{cm}^{2}$ for four temperatures[31]. Both measured data points and calculated curves are given. The parameters change, compared to the parameter set given in Table 1 to fit the curve for $T=460 \mathrm{~K}$ are here:

$$
\begin{aligned}
\tau_{0} & =2 \mathrm{~h}, G_{\mathrm{t} 0}=9 e 12 \mathrm{~A}^{-1} \mathrm{~cm}^{-1} \mathrm{~s}^{-1}, \\
V_{0} & =4 \mathrm{at} . \%, E_{\mathrm{form}}=0.1 \mathrm{eV}, E_{j}=0.45 \mathrm{eV} .
\end{aligned}
$$

The curves for the other temperatures were obtained without changing these parameters.

Figure 11 gives resistance change curves, on $\mathrm{Al} / \mathrm{Si}$ samples, at $j=3 \mathrm{MA} / \mathrm{cm}^{2}$ for four temperatures[31]. Both measured data points and calculated curves are given. The parameters change, compared to the parameter set given in Table 1 , to fit the curve for $T=460 \mathrm{~K}$ are here:

$$
\begin{aligned}
\tau & =1.5 \mathrm{~h}, G_{\mathrm{to}}=5.3 \mathrm{e} 14 \mathrm{~A}^{-1} \mathrm{~cm}^{-1} \mathrm{~s}^{-1}, \\
V_{0} & =4 \text { at. } \%, E_{\text {form }}=0.6 \mathrm{eV}, E_{j}=0.6 \mathrm{eV} .
\end{aligned}
$$

The curves for the other temperatures were obtained without changing these parameters.

The results in Figs 10 and 11 show the capability of the model in describing resistance change curves as a function of temperature.

The temperature dependence of the exponential resistance increase part of the curves is described by the temperature dependence in the current densityinduced formation (through $E_{j}$ ). The temperature dependent remanence of resistance change is ac-

Table 2. Comparison between activation energies from model calculations and activation energies derived directly from the resistance change measurements

\begin{tabular}{llll}
\hline Activation energy (eV) & & $\mathrm{Al}$ & $\mathrm{Al} / \mathrm{Si}$ \\
\hline$V_{0}=V_{10} \exp \left(-E_{\text {form }} / k T\right)$ & $E_{\text {form }}$ & 0.1 & 0.6 \\
$G(j)=\left.G_{\mathrm{t0}} b\right|^{m} \exp \left(-E_{j} / k T\right)$ & $E_{j}$ & 0.45 & 0.6 \\
$E_{\mathrm{a}}(\delta R)$ taken from $[31]$ & $E_{\mathrm{a}}$ & 0.43 & 0.56 \\
\hline
\end{tabular}

counted for by the model through the thermal vacancy equilibrium concentration [refer to eqn (5)]. The amount of remanence depends on the magnitude of the redistribution of the imperfections (refer to Section 3.4). This again is related to the magnitude of the vacancy flux [refer to eqn (18)], which scales with the thermal equilibrium vacancy concentration. The calculated resistance change curves are therefore relatively insensitive for the value of $\rho_{\mathrm{gb}}$, since these curves show no linear increase part and the temperature dependent magnitude of the remanence is accounted for by the temperature dependent thermal vacancy equilibrium concentration ( $\rho_{g b}$ is assumed to be temperature independent).

In Table 2 the activation energies $E_{\text {form }}$ and $E_{j}$ obtained from the model calculations to match the measured resistance change curves and the activation energies $\left(E_{\mathrm{a}}\right)$ derived from the resistance changes by Kraayeveld[31] are given. The activation energy for the current density-induced excess formation, $E_{j}$, shows a very close agreement with the activation energy, $E_{\mathrm{a}}$, directly derived from the resistance changes. The formation energy, $E_{\mathrm{form}}$, for aluminium is found to be much less than that obtained for $\mathrm{Al} / \mathrm{Si}$, and this activation energy differs from the current density-induced formation activation energy and the activation energy obtained from the resistance changes. This is not the case for $\mathrm{Al} / \mathrm{Si}$. The negligible temperature dependence of the remanence of the resistance change for the curves in Fig. 10 results in such a low (and inaccurate) value for the activation energy. It is not clear from the single set of measurements whether this behaviour is typical for aluminium.

Lloyd and Koch[11] report the activation energy for the formation of vacancies in $\mathrm{Al}$ to be on the order of $0.5 \mathrm{eV}$, this agrees well with the activation energy $E_{\mathrm{a}}$ of the resistance change and the activation energy, $E_{j}$, for the current density-induced excess formation.

\section{DISCUSSIONS}

From our experimental verification we observe a difference in $\tau_{0}$ for $\mathrm{Al}$ and $\mathrm{Al} / \mathrm{Si}$. In all cases $\tau_{0}$ is found to be smaller for $\mathrm{Al} / \mathrm{Si}$ than for $\mathrm{Al}$. From our model point of view this implies the vacancy lifetime to be higher in $\mathrm{Al}$ than in $\mathrm{Al} / \mathrm{Si}$. It is difficult to give a physical explanation for this at the moment; more measurements are required. This difference in $\tau_{0}$ implies that the build-up of vacancies is faster in $\mathrm{Al} / \mathrm{Si}$ than in $\mathrm{Al}$. The magnitude of $\tau_{0}$ is directly related to the exponential changes in the resistance change curves. Its value in the order of hours seems very long for a vacancy lifetime; it may very well represent a some more complicated phenomenon. On the other hand, known estimates for vacancy lifetimes are based on bulk materials; vacancy lifetimes can very well be orders in magnitude higher in grain boundary regions. 
Under equal stressing conditions the magnitude of resistance change is less for $\mathrm{Al}$ than for $\mathrm{Al} / \mathrm{Si}$. This shows in the model parameter $G_{0}$; this parameter is less for $\mathrm{Al}$ than $\mathrm{Al} / \mathrm{Si}$. The parameter $G_{0}$ can be considered as a representation of the sensitivity of the material for the current density-induced excess formation of vacancies.

The resistivity in the grain boundary, $\rho_{\mathrm{gb}}$, gives a measure of the sensitivity of a material for the creation of imperfections, thus may give a representation of the sensitivity of the material for structural damage formation. A higher $\rho_{\mathrm{gb}}$ results in a steeper slope of the linear part of the resistance change curve, in turn giving rise to a higher remanence.

The model calculations on the complete resistance change curves (exponential, linear increase and exponential decay with remanence, Fig. 8) show that the amount of remanence directly related to the linear increase part of the resistance change (which is inherent to the model) also holds for the expcriments. The resistance change curves in Fig. 8 show the spread in resistance change between two macroscopically identical samples. The cause for this has to be a difference in microstructure of the films. In terms of our model these microstructural differences refiect into changing two parameters, $G_{0}$ and $\rho_{\mathrm{gb}}$. Even though the resistance change difference between the curves is about $50 \%$, the model parameter changes required to describe these curves are only about $10-20 \%$. This is in agreement with experiments, where a wide spread of resistance change curves can be found for macroscopically identical samples.

The current density-induced formation, $G(j)$, is necessary in order to account for the experimentally observed current density dependence of the resistance change, although the physical background is not quite clear. The magnitude of the current densityinduced formation is small compared to the thermal equilibrium concentration $V_{0}$. In all of the curves calculated in the experimental verification (Section 4) $\tau_{0} G(j)$ is in the order of $10^{-3} V_{0}$. In pure single crystalline aluminium current density-induced formation seems an unlikely process. However in ICmetallization the layers are poly-crystalline with many dislocations and imperfections. Also in $\mathrm{Al} / \mathrm{Si}$ we can have silicon precipitates disturbing the crystal lattices. This makes the current density-induced formation term plausible. We already see that the buildup of vacancies in $\mathrm{Al} / \mathrm{Si}$ is faster than in $\mathrm{Al}$, supporting this concept.

For the sake of simplicity a homogeneous formation is used so far. We have achieved similar resistance change simulation results with a physically more plausible grain boundary-dependent current density-induced formation with some adjustments to the grain boundary parameter $\rho_{\mathrm{gb}}$ (and/or $w_{\mathrm{gb}}$ ).

The capability of the model in extracting parameters that may help in gaining more insight in the process of electromigration is shown. One important result of the parameter extraction is that the current density-induced formation is linearly dependent on the current density $(m=1)$. Also clear differences in parameter values are obtained for different conductor materials, but more measurements are required before significant conclusions can be drawn on this.

It should be noted that even in the very early stages of electromigration more than just a redistribution of vacancies takes place. The accumulation of damage, in the form of imperfections, occurs at every current density level in sample. The available measurements do not require any electromigration threshold current density in the model.

\section{CONCLUSIONS}

An empirical model for electromigration-induced early resistance changes has been introduced. The major features of this model are the use of two continuity equations, both with formation and annihilation: one for the vacancies (mobile) and one for the imperfections (immobile). This, under current stress, results in a steady-state vacancy distribution and an imperfection distribution which continues to change in time. The resistance change modelled, in terms of the behaviour of the redistribution of vacancies and imperfections, can physically be interpreted as caused by structural changes occurring mainly in and near the grain boundaries.

The model uses a small, but essential current density-induced formation term. This current density-induced formation term has been shown to be linearly dependent on the current density.

The model is capable of simulating the following early resistance change characteristics:

- exponential resistance increases followed by linear increases,

- resistance changes as a function of the applied current density,

- resistance changes as a function of the temperature,

- exponential decay towards a non-zero resistance change (remanence).

The for the model inherent relation between the linear increase and the remanence of resistance change corresponds very well with the experiments. Slight parameter changes can account for a large spread in resistance change curves.

Only three model-parameters $\left(\rho_{\mathrm{gb}}, G_{0}, \tau_{0}\right)$ are used to fit the early resistance change curves; all other model parameters have generally accepted values. The model can very well be used as a starting point in the development of a reliability circuit simulator.

\section{REFERENCES}

1. P. J. Marcoux, P. P. Merchant, V. Naroditski and W. D. Rehder, A new 2D simulation model of electromigration. Hewlett-Packard J. (1989). 
2. R. Kirchheim and U. Kaeber, J. appl. Phys. 70, 172 (199I).

3. M. J. Attardo, R. Rutledge and R. C. Jack, J. appl. Phys. 42, No. 11 (1971).

4. J. M. Schoen, J. appl. Phys. 51, No. 1 (1980).

5. J. Cho and C. V. Thompson, Appl. Phys. Lett. 54, No. 25 (1989).

6. J. W. Harrison Jr, IEEE Trans. Electron Devices 35, No. 12 (1988).

7. M. Shatzkes and J. R. Lloyd, J. appl. Phys. 59, No. 11 (1986).

8. E. Castaño, J. Maiz, P. Flinn and M. Madden, Appl. Phys. Lett. 59, 129 (1991).

9. P. R. Besser, M. C. Madden and P. A. Flinn, In situ observations of the dynamic behavior of electromigration voids in passivated aluminum lines. Private communication.

10. M. Madden, T. Marieb, E. Abratowski and P. A. Flinn, High resolution observation of void motion in passivated metal lines under electromigration stress. In $\mathrm{Ma}$ terials Reliability in Microelectronics II (edited by C. V. Thompson and J. R. Lloyd), pp. 33-38. Mater. Res. Soc. 265, Pittsburgh, Pa (1992).

11. J. R. Lloyd and R. H. Koch, Proc. IRPS, p. 161 (1987).

12. K. Hinode, T. Furusawa and Y. Homma, Proc. IRPS, p. 205 (1992).

13. J. Niehof, P. A. Flinn and T. J. Maloney, Proc. ESREF 92, pp. 359-362 (1992) and QRE Int. 9, 295 (1993).

14. J. R. Kraayeveld, A. H. Verbruggen and S. Radelaar, Comparison between $1 / f$ noise, high resolution resistometric, and lifetime electromigration studies of $\mathrm{Al}$ and Al(Si), Mater. Res. Soc. Symp. Proc., Materials reliability in microelectronics III, San Francisco (1993).

15. U. Möckl, Untersuchung der Electromigration an passivierten Al-1\%Cu-Leiterbahnen mittels hochauflösender Widerstandsmessung. Diplom Arbeit Univ. of Stuttart and Max Planck Institute für Metallforschung (1992).
16. J. Niehof, H. C. de Graaff and J. F. Verwey, Empirical modelling of electromigration early resistance changes, Mater. Res. Soc. Symp. Proc., Materials reliability in microelectronics III, San Francisco (1993).

17. Z. Li, C. L. Bauer and S. Mahajan, Appl. Phys. Lett. 61, No. 3 (1992).

18. A. Scorzoni, G. C. Cardinaly, G. L. Baldini and G. Soncini, Microelectron. Rel. 30, No. 1, (1990).

19. S. Shingubara, H. Kaneko and M. Saitoh, J. appl. Phys. 69, No. 1 (1991).

20. J. Niehof, Electromigration early resistance change modelling. Ph.D. thesis, Univ. of Twente, The Netherlands (1994).

21. J. Niehof, H. C. de Graaff and J. F. Verwey, Mater. Res. Soc. Symp. Proc. 338, San Francisco (1994).

22. B. K. Liew, N. W. Cheung and C. Hu, Proc. IRPS p. 215 (1989).

23. B. K. Liew, Modelling and testing of electromigration effects in integrated circuit interconnects and contacts under time-varying current conditions. Ph.D. dissertation, Univ. of California at Berkeley (1990).

24. A. S. Nandedkar, Mater. Res. Soc. Symp. Proc., Fall Meeting Symp. O (1992).

25. Ph. B. M. Wolbert, Modelling and simulation of semiconductor devices in TRENDY, electrical thermal and hydrodynamic behavior. Ph.D. thesis, Univ. of Twente, The Netherlands (1991).

26. A. Scorzoni, I. de Munari and H. Stulens, Mater. Res. Soc. Symp. Proc., Advanced Metallization for Devices and Circuits-Science, Technology and Manufacturability 111,337 (1994).

27. R. Kirchheim, Acta metall. mater. 40, 309 (1992).

28. R. Kirchheim, Mater Res. Soc. Symp. Proc. 309, 101 (1993).

29. B. K. Liew, P. Fang, N. W. Cheung and C. Hu, Proc. IRPS, p. 111 (1990).

30. J. R. Kraayeveld, Private communication (1993).

31. J. R. Kraayeveld, A. H. Verbruggen and S. Radelaar, Mater. Res. Soc. Symp. Proc. 309, 307 (1993). 